\title{
Flora intestinal, probióticos, prebióticos, simbióticos y alimentos novedosos
}

\author{
A. S. Peña \\ Inmmunogenética. Departamento de Patología. Centro Médico Universitario VU. Amsterdam, Países Bajos
}

Peña AS. Flora intestinal, probióticos, prebióticos, simbióticos y alimentos novedosos. Rev Esp Enferm Dig 2007; 99: 653-658.

\section{INTRODUCCIÓN}

Las observaciones realizadas en modelos animales de experimentación y en seres humanos indican que la flora intestinal supone una gran cantidad de microorganismos comensales que han evolucionado en armonía con su huésped y mejorado la salud de este último (1). Estas bacterias intervienen en el desarrollo normal del sistema inmunitario y en la regulación de la respuesta a los patógenos, y son esenciales para el establecimiento y el mantenimiento de la tolerancia inmunitaria de la mucosa (26). La flora intestinal participa en varios procesos fisiológicos, como la digestión y la motilidad $(7,8)$ además de en funciones metabólicas del organismo tales como la producción de vitaminas. También aporta a los colonocitos sustratos como el butirato (9). El ácido butírico y el butirato presentes en la luz del colon tras la digestión de, por ejemplo, alimentos ricos en fibra, regulan la diferenciación de las células mucosas del intestino grueso e inducen la apoptosis, que es importante para controlar la inflamación y evitar la aparición del cáncer $(10,11)$.

\section{LA IMPORTANCIA DE LA UBICUA FLORA INTESTINAL EN LA PREVENCIÓN DE ENFERMEDADES}

Revisaremos las evidencias disponibles qe demuestran que la flora intestinal protege al hombre de enfermedades

Recibido: 30-08-07.

Aceptado: 08-10-07.

Correspondencia: A. S. Peña. Inmunogenética. Departamento de Patología. VUmc (VU University Medical Centre). De Boelelaan 1117. 1081 HV Amsterdam, Países Bajos. Fax: 0031204444 737.e-mail: as.pena@vumc.nl como el asma, la alergia y las enteropatías inflamatorias crónicas, así como los mecanismos moleculares que se conocen. Ahora es posible realizar estudios científicos sobre este tema gracias a las nuevas tecnologías que permiten estudiar las muestras fecales humanas mediante protocolos de PCR que no precisan cultivos. Hoy también podemos detectar e identificar las bacterias predominantes mediante cebadores específicos de grupo dirigidos al ARNr de $16 \mathrm{~S}$ y polimorfismos de la longitud del fragmento de restricción terminal. Esta tecnología permite estudiar la composición y la dinámica de la microflora intestinal sin necesidad de recurrir al cultivo de las heces que habitualmente tardan demasiado tiempo en conocerse los resultados (12-14).

Estos nuevos adelantos tecnológicos van acompañados de avances en el conocimiento de las bases de la simbiosis huésped-microorganismo y de la interacción de la microflora intestinal con la inmunidad innata y adquirida o adaptada.

\section{LAS BASES MOLECULARES DE LA SIMBIOSIS HUÉSPED-MICROORGANISMO}

Uno de los avances clave que estimularán aun más las investigaciones y que contribuirán a resolver el dilema de si usar las cepas de bacterias aisladas o las mezclas de distintas cepas en la regulación de la inflamación intestinal (15), los simbióticos (16-20), los péptidos antimicrobianos catiónicos (21) o los polisacáridos capsulares zwitteriónicos (PSZ), es el mayor conocimiento de las bases moleculares de la simbiosis huésped-microorganismo (22).

Las recientes investigaciones sobre una de las moléculas arquetípicas de las bacterias comensales que median en el desarrollo del sistema inmunitario del huésped (23), indican que los PSZ son capaces de modular el complejo ecosistema del tubo digestivo. Bajo esta perspectiva, di- 
cho conocimiento nos ayuda ahora a comprender viejas observaciones que, no obstante, siguen siendo relevantes hoy en día. Strachan, de la School of Hygiene and Tropical Medicine de Londres propuso en 1989 (24) que cambios en la flora bacteriana serían los responsables de la mayor incidencia de trastornos inmunitarios tales como la alergia y la atopia. Actualmente lo llamamos la hipótesis higiénica (25), aunque aún se desconoce si la ausencia de bacterias comensales como los lactobacilos y/o las bifidobacterias predispone o no al individuo a padecer la enfermedad inflamatoria intestinal. Por ejemplo, existen pruebas de que el Lactobacillus plantarum induce la síntesis y secreción de la citocina antiinflamatoria IL-10 en los macrófagos y las células T que provienen del colon inflamado (26). La flora endógena intestinal es capaz de sintetizar PSZ. Estas moléculas bacterianas emplean el sistema de presentación del complejo mayor de histocompatibilidad (CMH) II para activar las células T mediante su reconocimiento por las proteínas receptoras alfa/beta de dichas células T (27-29). Las observaciones decisivas que aportaron pruebas de la importancia biológica que tiene la activación de las células $\mathrm{T}$ por estos polímeros zwitteriónicos no se realizaron hace mucho. Células T CD4+ humanas estimuladas con estas moléculas in vitro y transferidas a ratas in vivo confirieron protección frente a los abscesos intraabdominales inducidos con un estímulo bacteriano viable $(30,31)$.

\section{INMUNIDAD INNATA}

Los avances realizados sobre la inmunidad innata y su relación con la inmunidad adquirida están permitiendo conocer mejor los mecanismos que controlan la inflamación en el tubo digestivo. Su identificación y tipificación funcional, incluido el descubrimiento de mutantes que bloquean totalmente la transducción de señal del NFKB, como los receptores extracelulares parecidos a Toll (TLR) y los receptores intracelulares NOD/CARD, están aportando nuevos datos con que comprender las relaciones existentes entre las bacterias intestinales y el huésped, y entre las bacterias intestinales y la enfermedad clínica.

\section{PROTEÍNAS RELACIONADAS CON TOLL}

Las proteínas relacionadas con Toll, de las que se han identificado unas 12 hasta la fecha, aparecen muy conservadas a lo largo de la evolución. Los TLR se expresan tanto en los enterocitos como en las células inmunitarias. Reconocen determinados componentes microbianos a través de los dominios de repeticiones ricas en leucina (LRR), como determinantes de superficie, el lipopolisacárido (LPS) de las bacterias gramnegativas (TLR2 y TLR4) y las secuencias CpG no metiladas del ADN (TLR9). Su activación induce la producción de citocinas de tipo T cooperador 1 (Th1) a través de un proceso de- pendiente de la activación del NFKB $(32,33)$. El LPS y la fracción lipídica A del LPS son reconocidos por el TLR4. En el ser humano, la mutación D299G, que afecta al dominio LRR del TLR4, se asocia a una respuesta menos aguda al LPS inhalado. La mayor expresión de este receptor en las células epiteliales de los pacientes con enteropatías inflamatorias se ha relacionado con los cambios en la flora intestinal (34).

\section{FAMILIA DE PROTEÍNAS NOD-LRR}

Otros productos de la flora bacteriana intestinal, como el peptidoglicano de las bacterias grampositivas, son capaces de estimular unos receptores específicos, la familia de proteínas NOD. Se trata de receptores citoplasmáticos que también se caracterizan por la presencia de LRR. Los receptores NOD1 y NOD2 son capaces de estimular el factor de transcripción NFKB. La presencia de regiones activadoras de caspasa en las proteínas NOD indica su importancia en la apoptosis (35).

\section{INMUNIDAD ADQUIRIDA, EL COMPONENTE CELULAR T}

En circunstancias normales, la respuesta inmunitaria intestinal a las bacterias residentes se verá limitada por una respuesta inmunitaria supresora (la llamada respuesta $\mathrm{TH} 2$ ) con predominio de IgA e IL-10. Hallazgos recientes han revelado que determinadas células $\mathrm{T}$ reguladoras, como las Th3, que producen factor transformador del crecimiento $\beta$ (TGF- $\beta$ ), y las células Tr 1 , que producen IL10 , regulan la respuesta inflamatoria de la mucosa. El déficit de citocinas o tipos celulares conduce a inflamación de la mucosa a causa de la respuesta anormal a la flora endógena intestinal. Se ha observado que los lactobacilos previenen la aparición de colitis espontánea en los ratones con déficit de interleucina 10 (36) y que la aportación continuada de Lactobacillus plantarum atenúa la inflamación en este modelo (37).

\section{ENFERMEDADES DE BARRERA}

La enfermedad de Crohn y la colitis ulcerosa son procesos inflamatorios crónicos de tipo autoinmunitario que afectan al tracto gastrointestinal. A menudo se afectan otros órganos, como los ojos, la piel y las articulaciones. La EII puede acompañarse de otras enfermedades de origen autoinmunitario. Avances recientes en la genética y los mecanismos moleculares de las proteínas que codifican estos genes han dado lugar a un nuevo panorama en el conocimiento de estas enfermedades complejas. La activación de genes específicos que afectan a la presentación de antígenos y el manejo de las células por la inmunidad innata pueden generar autoinmunidad, con la consiguiente activa- 
ción del complejo mayor de histocompatibilidad (CMH) y de las múltiples citocinas que están implicadas en la regulación de la inmunidad adquirida.

La mejor forma de clasificar esta constelación de enfermedades es como enfermedades de barrera, probablemente debidas a la falta de adaptación del sistema inmunitario innato al medio y a la "occidentalización" de la civilización (38). Estas enfermedades afectan a 1-5 de cada 1.000 individuos y suponen una gran carga para los sistemas nacionales de salud de muchos países de continentes distintos. A escala mundial, uno de los grandes retos sería generar intervenciones que evitaran la aparición de estas enfermedades en Asia, América Latina y Africa (39).

\section{INMUNIDAD INNATA Y ENFERMEDAD DE CROHN}

\section{Las proteínas NOD}

Los estudios han demostrado que el gen que codifica NOD2, el gen $C A R D 15$, es el primer gen implicado en la susceptibilidad a la enfermedad de Crohn. La NOD2 se expresa también en las células del epitelio intestinal y las de Paneth. Las mutaciones del gen CARD15 pueden ser un componente clave de las respuestas innatas anormales de la mucosa a las bacterias de la luz intestinal. Por tanto, el fallo de esta interacción podría contribuir al desarrollo de la enfermedad de Crohn (40). Aunque estas mutaciones dependen de la ecología microbiológica de una población y, por tanto, su frecuencia varía en las distintas poblaciones. Esto probablemente explique que en las poblaciones asiáticas (41-44) y africanas (45) no se hayan encontrado diferencias significativas en cuanto a portadores de las mutaciones de CARD15 entre los pacientes con enfermedad de Crohn y los controles.

\section{Defensinas alfa y beta}

NOD1 y NOD2 parecen tener actividad bactericida al modular la producción epitelial de defensinas, lo que explica la reducida expresión de defensinas alfa en el íleon de los pacientes con enfermedad de Crohn $(46,47)$.

Las defensinas beta humanas (HBD-2) están disminuidas en el colon de pacientes con enfermedad de Crohn (48). El aumento de las HBD-2 en la colitis ulcerosa puede mejorar con el uso de simbióticos (49) y de E. coli Nissle, que se ha visto que mantiene la remisión de la colitis ulcerosa (50) e induce la expresión de HBD-2 (51).

El número de copias de $\mathrm{ADN}$ del complejo del gen de la defensina beta en el cromosoma 8p23.1 es muy polimorfo en la población sana, y hallazgos recientes indican que un número menor de copias del gen HBD-2 en el locus de la defensina beta predispone a la colitis de Crohn, lo más probable es que a través de una menor expresión de defensina beta (52). En consecuencia, las localizaciones regionales de la EC, la afetación ileal o cólica, pueden vincularse a defectos diferentes en la expresión de defensinas (53).

\section{PROBIÓTICOS, PREBIÓTICOS, SIMBIÓTICOS, ALIMENTOS NUEVOS O NOVEDOSOS}

Los probióticos afectan de manera beneficiosa al huésped al mejorar las propiedades de la microflora indígena. Los probióticos también son importantes para la maduración de la inmunidad humoral en la primera infancia $(54,55)$. Son útiles para tratar y prevenir la diarrea infecciosa aguda de lactantes y niños (56); esto se ha observado en ensayos aleatorizados, doble ciego y controlados con placebo $(57,58)$. El uso profiláctico de la administración oral de Lactobacillus $G G$ redujo significativamente el riesgo de diarrea nosocomial en los lactantes, especialmente el de la gastroenteritis nosocomial por rotavirus (59). Una revisión sistemática de Cochrane en 2004 concluyó que los probióticos parecen ser útiles como adyuvantes de la rehidratación para tratar la diarrea infecciosa aguda de adultos y niños. Se necesitan más investigaciones para documentar el uso de pautas específicas de probióticos en grupos de pacientes concretos (60). Recientemente se ha observado que el Saccharomyces boulardii es beneficioso en la diarrea asociada a antibióticos (61).

Los prebióticos, como los fructanos de tipo inulínico, se ha visto que mejoran las funciones metabólicas de la flora comensal. Datos clínicos y experimentales indican que mejoran la barrera mucosa digestiva y modulan las funciones tróficas de la flora. Los prebióticos también podrían ayudar a prevenir las enfermedades inflamatorias del intestino (62).

Parece que la justificación del uso de los simbióticos, es decir, de los productos resultantes de la combinación de probióticos y prebióticos, se basa en observaciones que muestran que la mejoría de la supervivencia de las bacterias probióticas durante el tránsito por el tracto digestivo superior. La implantación más eficiente en el colon y el efecto estimulante del crecimiento de los probióticos y la flora bacteriana intestinal contribuyen a mantener la homeostasis intestinal y la salud del organismo. Los japoneses introdujeron el término "alimentos funcionales", y en Europa se está usando el de "alimentos nuevos o novedosos". Indica que ciertos componentes podrían contribuir a reducir la presión arterial o los niveles de colesterol mediante el uso de fitoesteroles. Sin embargo, no existen datos referentes al uso de estos nutrientes en el tratamiento de la EII.

\section{JUSTIFICACIÓN DEL USO DE PROBIÓTICOS EN LA ENFERMEDAD INFLAMATORIA INTESTINAL}

A pesar de los efectos terapéuticos y profilácticos de los probióticos, estos siguen sin formar parte del tratamiento habitual de las enfermedades inflamatorias del intestino o de los trastornos de la motilidad del tubo diges- 
tivo. En la colitis ulcerosa, la inflamación queda limitada a la mucosa y la submucosa del colon; el síntoma de presentación más frecuente es la diarrea sanguinolenta. En la enfermedad de Crohn, la inflamación puede afectar a la pared intestinal y cualquier parte del tubo digestivo. Es característico que las zonas de inflamación estén en continuidad con zonas de mucosa normal.

Los avances en la patogenia de la inflamación intestinal tanto aguda como crónica indican que los probióticos, los prebióticos y/o los simbióticos resultarán útiles en el tratamiento de estos trastornos. El uso de la cepa Shirota de Lactobacillus casei ha llevado a mejorar la enteropatía inflamatoria crónica del ratón y se asocia a una regulación a la baja de la producción de IL-6 e IFN-gamma en la lámina propia de las muestras colónicas (63).

Una mezcla de varias cepas probióticas ha mostrado que ejerce efectos beneficiosos en el tratamiento de la colitis ulcerosa leve (64-66), en el tratamiento de la reservoritis y el mantenimiento de la remisión $(67,68)$. Los estudios de pacientes con reservoritis en remisión mediante PCR en tiempo real han mostrado que el VSL\#3 aumenta el número total de células bacterianas $(\mathrm{p}=0,002)$ y modifica el espectro de bacterias en favor de las especies anaeróbicas. Las bibliotecas de clones específicos de Lactobacilli y Bifidobacteria mostraron que la riqueza y el espectro de estas bacterias se alteraban con el tratamiento probiótico. Restablecer la integridad de una mucosa intestinal mediante una flora intestinal "protectora" podría ser por tanto uno de los posibles mecanismos que dan lugar a los efectos beneficiosos de las bacterias probióticas en las enfermedades de la barrera intestinal del tubo digestivo inferior (69).

\section{EFECTOS BENEFICIOSOS EN OTRAS INDICACIONES}

La seguridad y la eficacia de VSL\#3 en pacientes con EII quiescente que habían presentado artralgia durante más de dos semanas se estudió recientemente en un ensayo abierto (70). Según estas observaciones clínicas preliminares, proponemos la hipótesis de que los probióticos podrían ser útiles en el tratamiento de las manifestaciones extraintestinales de la enfermedad inflamatoria intestinal como la artralgia en los pacientes con colitis ulcerosa y enfermedad de Crohn (71). Sin embargo, se necesitan estudios aleatorizados y controlados que confirmen estos efectos.

Hoy disponemos de resultados prometedores de los probióticos en el tratamiento del síndrome del intestino irritable $(72,73)$.

\section{EFECTOS BENEFICIOSOS DE LOS SIMBIÓTICOS}

Aún no son muchos los estudios que han examinado los efectos de los simbióticos sobre el sistema inmunita- rio de la mucosa intestinal. Estudios recientes indican que los probióticos podrían inhibir la activación del NFKB en los linfocitos aislados de la lámina propia de las muestras de biopsia intestinal y regular a la baja la secreción de citocinas inflamatorias en los tejidos inflamados de los pacientes con colitis ulcerosa $(74,75)$.

Las observaciones recientes sobre el uso de simbióticos en la colitis ulcerosa son estimulantes $(49,76)$. Un ensayo aleatorizado y controlado con placebo, realizado en Japón sobre 20 pacientes con colitis ulcerosa utilizando $100 \mathrm{ml} /$ día de suplemento de leche fermentada con bifidobacterias o de placebo durante 12 semanas, mostró que el índice de actividad clínica, endoscópica e histológica era significativamente menor en el grupo que recibió leche fermentada por bifidobacterias que en el grupo del placebo después del tratamiento. Los aumentos de las concentraciones fecales de butirato, propionato y ácidos grasos de cadena corta fueron significativos en el grupo tratado con leche fermentada por bifidobacterias, pero no en el grupo del placebo (77).

Una ventaja adicional que supone el uso de los simbióticos es su falta de patogenicidad incluso en pacientes inmunocomprometidos y su seguridad en niños y adultos. Aunque las cepas usadas para los probióticos se eligen a partir de la flora comensal de los seres humanos y no presentan ninguna resistencia intrínseca a los antibióticos, se debe mantener una vigilancia que detecte los posibles casos raros de infección por probióticos debe mantenerse. En este caso, deben enviarse los aislados a los centros de referencia para su caracterización molecular y confirmación.

\section{EL RETO PARA EL FUTURO}

El reto para los expertos que trabajan en el uso médico de los alimentos funcionales y en el campo de los probióticos, prebióticos, simbióticos y alimentos nuevos consiste en aplicar los nuevos conocimientos que generan los científicos básicos en el campo de la flora intestinal y del desarrollo de simbióticos para mejorar el tratamiento de la alergia, la atopia y las enteropatías inflamatorias, y posiblemente prevenirlas.

Todos podemos estar de acuerdo con la declaración realizada por Bohm y Kruis (78): "La investigación probiótica, en la intersección de la gastroenterología con la inmunología y la microbiología, es muy dinámica en los campos tanto básico como clínico. Seguir conociendo los complejos mecanismos moleculares que conducen a la eficacia de los probióticos estimulará también el desarrollo de formulaciones probióticas de mayor éxito".

Pocas de las indicaciones que manejan los gastroenterólogos prácticos están basadas en pruebas. El desarrollo de una nueva tecnología para valorar el efecto que las distintas cepas de probióticos ejercen, solas o combinadas, sobre la modificación de la flora intestinal, y el papel que estos cambios desempeñan en el control de la infla- 
mación intestinal, permite predecir que los probióticos intervendrán un día de forma definitiva en el tratamiento de las enfermedades gastrointestinales.

\section{AGRADECIMIENTOS}

Esta revisión se basa en una ponencia presentada en el $1^{\text {er }}$ Congreso Internacional sobre Uso Médico de Alimentos Funcionales, celebrado en Tokio, Japón, el 17 de noviembre de 2006. La ponencia se llamaba: Flora intestinal y simbióticos. El autor agradece la beca de Yakult Honsha, Japón, para poder asistir al $1^{\text {er }}$ Congreso Internacional sobre Uso Médico de Alimentos Funcionales y desea dar las gracias al Comité Científico de la Sociedad Japonesa de Alimentos Funcionales por invitarme a dirigirme a sus miembros.

\section{BIBLIOGRAFÍA}

1. Ley RE, Peterson DA, Gordon JI. Ecological and evolutionary forces shaping microbial diversity in the human intestine. Cell 2006; 124 : 837-48.

2. Underhill DM, Ozinsky A, Hajjar AM, Stevens A, Wilson CB, Bassetti $\mathrm{M}$, et al. The Toll-like receptor 2 is recruited to macrophage phagosomes and discriminates between pathogens. Nature 1999; 401: 811-5.

3. Aderem A, Ulevitch RJ. Toll-like receptors in the induction of the innate immune response. Nature 2000; 406: 782-7.

4. Hemmi H, Takeuchi O, Kawai T, Kaisho T, Sato S, Sanjo H, et al. A Toll-like receptor recognizes bacterial DNA. Nature 2000; 408: 7405 .

5. Hayashi F, Smith KD, Ozinsky A, Hawn TR, Yi EC, Goodlett DR, et al. The innate immune response to bacterial flagellin is mediated by Toll-like receptor 5. Nature 2001; 410: 1099-103.

6. Yamamoto M, Sato S, Hemmi H, Sanjo H, Uematsu S, Kaisho T, et al. Essential role for TIRAP in activation of the signalling cascade shared by TLR2 and TLR4. Nature 2002; 420: 324-9.

7. Massi M, Ioan P, Budriesi R, Chiarini A, Vitali B, Lammers KM, et al. Effects of probiotic bacteria on gastrointestinal motility in guineapig isolated tissue. World J Gastroenterol 2006; 12: 5987-94.

8. Bazzocchi G, Gionchetti P, Almerigi PF, Amadini C, Campieri M. Intestinal microflora and oral bacteriotherapy in irritable bowel syndrome. Dig Liver Dis 2002; 34 (Supl. 2): S48-53.

9. Gassull MA. Review article: the intestinal lumen as a therapeutic target in inflammatory bowel disease. Aliment Pharmacol Ther 2006; 24 (Supl. 3): 90-5.

10. Collard TJ, Guy M, Butt AJ, Perks CM, Holly JM, Paraskeva C, et al. Transcriptional upregulation of the insulin-like growth factor binding protein IGFBP-3 by sodium butyrate increases IGF-independent apoptosis in human colonic adenoma-derived epithelial cells. Carcinogenesis 2003 ; 24: 393-401.

11. Giermasz A, Grzela T, Nowis D, Makowski M, Czajka A, Stoklosa $\mathrm{T}$, et al. Butyric acid enhances in vivo expression of hTNF-alpha in transduced melanoma cell line. Anticancer Res 2001; 21: 4001-4.

12. Brigidi P, Swennen E, Vitali B, Rossi M, Matteuzzi D. PCR detection of Bifidobacterium strains and Streptococcus thermophilus in feces of human subjects after oral bacteriotherapy and yogurt consumption. Int J Food Microbiol 2003; 81: 203-9.

13. Matsuki T, Watanabe K, Fujimoto J, Miyamoto Y, Takada T, Matsumoto K, et al. Development of 16S rRNA-gene-targeted groupspecific primers for the detection and identification of predominant bacteria in human feces. Appl Environ Microbiol 2002; 68: 5445-51.

14. Nagashima K, Hisada T, Sato M, Mochizuki J. Application of new primer-enzyme combinations to terminal restriction fragment length polymorphism profiling of bacterial populations in human feces.
Appl Environ Microbiol 2003; 69: 1251-62

15. Karimi O, Pena AS. Probiotics: Isolated bacteria strain or mixtures of different strains? Two different approaches in the use of probiotics as therapeutics. Drugs Today (Barc) 2003; 39: 565-97.

16. Rioux KP, Fedorak RN. Probiotics in the treatment of inflammatory bowel disease. J Clin Gastroenterol 2006; 40: 260-3.

17. Passeron T, Lacour JP, Fontas E, Ortonne JP. Prebiotics and synbiotics: two promising approaches for the treatment of atopic dermatitis in children above 2 years. Allergy 2006; 61: 431-7.

18. Bengmark S. Synbiotics and the mucosal barrier in critically ill patients. Curr Opin Gastroenterol 2005; 21: 712-6.

19. Sartor RB. Therapeutic manipulation of the enteric microflora in inflammatory bowel diseases: antibiotics, probiotics, and prebiotics. Gastroenterology 2004; 126: 1620-33.

20. Kanamori Y, Sugiyama M, Hashizume K, Yuki N, Morotomi M, Tanaka R. Experience of long-term synbiotic therapy in seven short bowel patients with refractory enterocolitis. J Pediatr Surg 2004; 39: 1686-92.

21. Peyrin-Biroulet L, Vignal C, Dessein R, Simonet M, Desreumaux P, Chamaillard M. NODs in defence: from vulnerable antimicrobial peptides to chronic inflammation. Trends Microbiol 2006; 14: 432-8.

22. Mazmanian SK, Kasper DL. The love-hate relationship between bacterial polysaccharides and the host immune system. Nat Rev Immunol 2006; 6: 849-58.

23. Mazmanian SK, Liu CH, Tzianabos AO, Kasper DL. An immunomodulatory molecule of symbiotic bacteria directs maturation of the host immune system. Cell 2005; 122: 107-18.

24. Strachan DP. Hay fever, hygiene, and household size. BMJ 1989; 299: 1259-60.

25. Anderson WJ, Watson L. Asthma and the hygiene hypothesis. N Engl J Med 2001; 344: 1643-4.

26. Pathmakanthan S, Li CK, Cowie J, Hawkey CJ. Lactobacillus plantarum 299: beneficial in vitro immunomodulation in cells extracted from inflamed human colon. J Gastroenterol Hepatol 2004; 19: 16673 .

27. Cobb BA, Kasper DL. Zwitterionic capsular polysaccharides: the new MHCII-dependent antigens. Cell Microbiol 2005; 7: 1398-403.

28. Stingele F, Corthesy B, Kusy N, Porcelli SA, Kasper DL, Tzianabos AO. Zwitterionic polysaccharides stimulate T cells with no preferential $\mathrm{V}$ beta usage and promote anergy, resulting in protection against experimental abscess formation. J Immunol 2004; 172: 1483-90.

29. Cobb BA, Wang Q, Tzianabos AO, Kasper DL. Polysaccharide processing and presentation by the MHCII pathway. Cell 2004; 117 : 677-87.

30. Tzianabos AO, Finberg RW, Wang Y, Chan M, Onderdonk AB, Jennings $\mathrm{HJ}$, et al. $\mathrm{T}$ cells activated by zwitterionic molecules prevent abscesses induced by pathogenic bacteria. J Biol Chem 2000; 275 : 6733-40.

31. Tzianabos AO, Chandraker A, Kalka-Moll W, Stingele F, Dong VM, Finberg RW, et al. Bacterial pathogens induce abscess formation by CD4(+) T-cell activation via the CD28-B7-2 costimulatory pathway. Infect Immun 2000; 68: 6650-5.

32. Korhonen R, Korpela R, Saxelin M, Maki M, Kankaanranta H, Moilanen E. Induction of nitric oxide synthesis by probiotic Lactobacillus rhamnosus GG in J774 macrophages and human T84 intestinal epithelial cells. Inflammation 2001; 25: 223-32.

33. Miettinen M, Lehtonen A, Julkunen I, Matikainen S. Lactobacilli and Streptococci activate NF-kappa B and STAT signaling pathways in human macrophages. J Immunol 2000; 164: 3733-40.

34. Cario E, Podolsky DK. Differential alteration in intestinal epithelial cell expression of toll-like receptor 3 (TLR3) and TLR4 in inflammatory bowel disease. Infect Immun 2000; 68: 7010-7.

35. Inohara N, Ogura Y, Fontalba A, Gutiérrez O, Pons F, Crespo J, et al. Host recognition of bacterial muramyl dipeptide mediated through NOD2. Implications for Crohn's disease. J Biol Chem 2003; 278 : 5509-12.

36. Madsen KL, Doyle JS, Jewell LD, Tavernini MM, Fedorak RN. Lactobacillus species prevents colitis in interleukin 10 gene-deficient mice. Gastroenterology 1999; 116: 1107-14.

37. Schultz M, Veltkamp C, Dieleman LA, Grenther WB, Wyrick PB, Tonkonogy SL, et al. Lactobacillus plantarum 299V in the treatment and prevention of spontaneous colitis in interleukin-10-deficient mice. Inflamm Bowel Dis 2002; 8: 71-80. 
38. Schreiber S, Rosenstiel P, Albrecht M, Hampe J, Krawczak M. Genetics of Crohn disease, an archetypal inflammatory barrier disease. Nat Rev Genet 2005; 6: 376-88.

39. Pena AS. Contribution of genetics to a new vision in the understanding of inflammatory bowel disease. World J Gastroenterol 2006; 12: 4784-7.

40. Hisamatsu T, Suzuki M, Reinecker HC, Nadeau WJ, McCormick BA, Podolsky DK. CARD15/NOD2 functions as an antibacterial factor in human intestinal epithelial cells. Gastroenterology 2003; 124: 993-1000.

41. Croucher PJ, Mascheretti S, Foelsch UR, Hampe J, Schreiber S. Lack of association between the C3435T MDR1 gene polymorphism and inflammatory bowel disease in two independent Northern European populations. Gastroenterology 2003; 125: 1919-20; author reply 1920-1.

42. Inoue N, Tamura K, Kinouchi Y, Fukuda Y, Takahashi S, Ogura Y, et al. Lack of common NOD2 variants in Japanese patients with Crohn's disease. Gastroenterology 2002; 123: 86-91.

43. Leong RW, Armuzzi A, Ahmad T, Wong ML, Tse P, Jewell DP, et al. NOD2/CARD15 gene polymorphisms and Crohn's disease in the Chinese population. Aliment Pharmacol Ther 2003; 17: 1465-70.

44. Sugimura M, Kinouchi Y, Takahashi S, Aihara H, Takagi S, Negoro $\mathrm{K}$, et al. CARD15/NOD2 mutational analysis in Japanese patients with Crohn's disease. Clin Genet 2003; 63: 160-2.

45. Zouiten-Mekki L, Zaouali H, Boubaker J, Karoui S, Fekih M, Matri S, et al. CARD15/NOD2 in a Tunisian population with Crohn's disease. Dig Dis Sci 2005; 50: 130-5.

46. Wehkamp J, Salzman NH, Porter E, Nuding S, Weichenthal M, Petras RE, et al. Reduced Paneth cell alpha-defensins in ileal Crohn's disease. Proc Natl Acad Sci U S A 2005; 102: 18129-34.

47. Wehkamp J, Schmid M, Fellermann K, Stange EF. Defensin deficiency, intestinal microbes, and the clinical phenotypes of Crohn's disease. J Leukoc Biol 2005; 77: 460-5.

48. Wehkamp J, Harder J, Weichenthal M, Mueller O, Herrlinger KR, Fellermann $\mathrm{K}$, et al. Inducible and constitutive beta-defensins are differentially expressed in Crohn's disease and ulcerative colitis. Inflamm Bowel Dis 2003; 9: 215-23.

49. Furrie E, Macfarlane S, Kennedy A, Cummings JH, Walsh SV, O'Neil DA, et al. Synbiotic therapy (Bifidobacterium longum/Synergy 1) initiates resolution of inflammation in patients with active ulcerative colitis: a randomised controlled pilot trial. Gut 2005; 54: 242-9.

50. Kruis W, Schutz E, Fric P, Fixa B, Judmaier G, Stolte M. Doubleblind comparison of an oral Escherichia coli preparation and mesalazine in maintaining remission of ulcerative colitis. Aliment Pharmacol Ther 1997; 11: 853-8.

51. Wehkamp J, Harder J, Wehkamp K, Wehkamp-von Meissner B, Schlee M, Enders C, et al. NF-kappaB- and AP-1-mediated induction of human beta defensin-2 in intestinal epithelial cells by Escherichia coli Nissle 1917: a novel effect of a probiotic bacterium. Infect Immun 2004; 72: 5750-8.

52. Fellermann K, Stange DE, Schaeffeler E, Schmalzl H, Wehkamp J, Bevins CL, et al. A chromosome 8 gene-cluster polymorphism with low human beta-defensin 2 gene copy number predisposes to Crohn disease of the colon. Am J Hum Genet 2006; 79: 439-48.

53. Wehkamp J, Stange EF. A new look at Crohn's disease: breakdown of the mucosal antibacterial defense. Ann N Y Acad Sci 2006; 1072: $321-31$.

54. Gronlund MM, Arvilommi H, Kero P, Lehtonen OP, Isolauri E. Importance of intestinal colonisation in the maturation of humoral immunity in early infancy: a prospective follow up study of healthy infants aged 0-6 months. Arch Dis Child Fetal Neonatal Ed 2000; 83: F186-92.

55. Gronlund MM, Salminen S, Mykkanen H, Kero P, Lehtonen OP. Development of intestinal bacterial enzymes in infants--relationship to mode of delivery and type of feeding. Apmis 1999; 107: 655-60.

56. Van Niel CW, Feudtner C, Garrison MM, Christakis DA. Lactobacillus therapy for acute infectious diarrhea in children: a meta-analysis. Pediatrics 2002; 109: 678-84.
57. Szajewska H, Mrukowicz JZ. Probiotics in the treatment and prevention of acute infectious diarrhea in infants and children: a systematic review of published randomized, double-blind, placebo-controlled trials. J Pediatr Gastroenterol Nutr 2001; 33 Suppl 2: S17-25.

58. Szajewska H, Mrukowicz JZ. Probiotics in prevention of antibioticassociated diarrhea: meta-analysis. J Pediatr 2003; 142: 85.

59. Szajewska H, Kotowska M, Mrukowicz JZ, Armanska M, Mikolajczyk W. Efficacy of Lactobacillus GG in prevention of nosocomial diarrhea in infants. J Pediatr 2001; 138: 361-5.

60. Allen SJ, Okoko B, Martinez E, Gregorio G, Dans LF. Probiotics for treating infectious diarrhoea. Cochrane Database Syst Rev 2004: CD003048.

61. Can M, Besirbellioglu BA, Avci IY, Beker CM, Pahsa A. Prophylactic Saccharomyces boulardii in the prevention of antibiotic-associated diarrhea: a prospective study. Med Sci Monit 2006; 12: PI19-22.

62. Guarner F. Inulin and oligofructose: impact on intestinal diseases and disorders. Br J Nutr 2005; 93 (Supl. 1): S61-5.

63. Matsumoto S, Hara T, Hori T, Mitsuyama K, Nagaoka M, Tomiyasu $\mathrm{N}$, et al. Probiotic Lactobacillus-induced improvement in murine chronic inflammatory bowel disease is associated with the down-regulation of pro-inflammatory cytokines in lamina propria mononuclear cells. Clin Exp Immunol 2005; 140: 417-26.

64. Guslandi M, Giollo P, Testoni PA. A pilot trial of Saccharomyces boulardii in ulcerative colitis. Eur J Gastroenterol Hepatol 2003; 15: 697-8.

65. Zocco MA, dal Verme LZ, Cremonini F, Piscaglia AC, Nista EC, Candelli M, et al. Efficacy of Lactobacillus GG in maintaining remission of ulcerative colitis. Aliment Pharmacol Ther 2006; 23: 156774.

66. Gionchetti P, Rizzello F, Lammers KM, Morselli C, Sollazzi L, Davies S, et al. Antibiotics and probiotics in treatment of inflammatory bowel disease. World J Gastroenterol 2006; 12: 3306-13.

67. Gionchetti P, Amadini C, Rizzello F, Venturi A, Campieri M. Treatment of mild to moderate ulcerative colitis and pouchitis. Aliment Pharmacol Ther 2002; 16 (Supl. 4): 13-9.

68. Gionchetti P, Rizzello F, Poggioli G, Morselli C, Lammers KM, Campieri M. Probiotic therapy to prevent pouchitis onset. Dis Colon Rectum 2005; 48: 1493; author reply 1493-4.

69. Kuhbacher T, Ott SJ, Helwig U, Mimura T, Rizzello F, Kleessen B, et al. Bacterial and fungal microbiota in relation to probiotic therapy (VSL\#3) in pouchitis. Gut 2006; 55: 833-41.

70. Karimi O, Peña AS, van Bodegraven AA. Probiotics (VSL\#3) in arthralgia in patients with ulcerative colitis and Crohn's disease: a pilot study. Drugs Today (Barc) 2005; 41: 453-9.

71. Karimi O, Peña AS. Probiotics in arthralgia and spondyloarthropathies in patients with inflammatory bowel disease. Prospective randomized trials are necessary. Rev Esp Enferm Dig 2005; 97: 570-4.

72. Camilleri M. Probiotics and irritable bowel syndrome: rationale, putative mechanisms, and evidence of clinical efficacy. J Clin Gastroenterol 2006; 40: 264-9.

73. Harris LA, Chang L. Irritable bowel syndrome: new and emerging therapies. Curr Opin Gastroenterol 2006; 22: 128-35.

74. Duncker SC, Lorentz A, Schroeder B, Breves G, Bischoff SC. Effect of orally administered probiotic E. coli strain Nissle 1917 on intestinal mucosal immune cells of healthy young pigs. Vet Immunol Immunopathol 2006; 111: 239-50.

75. Bai AP, Ouyang Q, Xiao XR, Li SF. Probiotics modulate inflammatory cytokine secretion from inflamed mucosa in active ulcerative colitis. Int J Clin Pract 2006; 60: 284-8.

76. Haskey N, Dahl WJ. Synbiotic therapy: a promising new adjunctive therapy for ulcerative colitis. Nutr Rev 2006; 64: 132-8.

77. Kato K, Mizuno S, Umesaki Y, Ishii Y, Sugitani M, Imaoka A, et al Randomized placebo-controlled trial assessing the effect of bifidobacteria-fermented milk on active ulcerative colitis. Aliment Pharmacol Ther 2004; 20: 1133-41.

78. Bohm SK, Kruis W. Probiotics: do they help to control intestinal inflammation? Ann N Y Acad Sci 2006; 1072: 339-50. 